\title{
A new MIR bow shock source in the Galactic center
}

\author{
N. Sabha ${ }^{1,2, \dagger, \ddagger}$, M. Zamaninasab ${ }^{2, \ddagger}$ A. Eckart ${ }^{1,2}$ and L. Moser ${ }^{1}$ \\ ${ }^{1}$ I.Physikalisches Institut, Universität zu Köln, Zülpicher Str.77, 50937 Köln, Germany \\ email: sabha@ph1.uni-koeln.de \\ ${ }^{2}$ Max-Planck-Institut für Radioastronomie, Auf dem Hügel 69, 53121 Bonn, Germany \\ ${ }^{\dagger}$ Member of Bonn Cologne Graduate School of Physics and Astronomy (BCGS) \\ ${ }^{\ddagger}$ Member of SFB 956 A2, funded by the Deutsche Forschungsgemeinschaft (DFG)
}

\begin{abstract}
We find a convex-like feature at a distance of $0.68 \mathrm{pc}\left(17^{\prime \prime}\right)$ from the position of the supermassive black hole, Sgr A*, at the center of the nuclear stellar cluster. This feature resembles a stellar bow shock with a symmetry axis pointing to the center. We discuss the possible nature of the feature and the implications of its alignment with other dusty comet-like objects inside the central parsec.
\end{abstract}

Keywords. Galaxy: nucleus — infrared: general — stars: winds, outflows

\section{Introduction}

The data we discuss here were obtained on the nights of 2010 September 11 and 13 at the ESO VLT using the MIR camera VISIR. We observed the Galactic center (GC) in two filters in the $N$-band, PAH1 $(8.59 \mu \mathrm{m}) \&$ NeII $(13.04 \mu \mathrm{m})$ with a pixel scale of $0.127^{\prime \prime}$ pixel $^{-1}$. Figure 1 shows a mosaic of $\sim 3 \times 3 \mathrm{pc}\left(74^{\prime \prime} \times 74^{\prime \prime}\right)$ that was constructed by combining 10 offset positions, each with $\sim 32.5^{\prime \prime} \times 32.5^{\prime \prime}$ FOV. Prominent features can be seen in the central parsec such as the minispiral (northern and eastern arm and parts of the western arc), several bright circumstellar dusty sources (IRS 1, 3, 5), IRS 13 cluster of stars and IRS 8 stellar bow shock (e.g. Tanner et al. 2005, Viehmann et al. 2006). Also visible in the mosaic is an extended feature that has a convex-like structure present at a distance of $0.68 \mathrm{pc}\left(17^{\prime \prime}\right)$ from the supermassive black hole (SMBH) Sgr A*. This feature resembles a stellar bow shock with a symmetry axis pointing to the north-east direction, i.e. the center of the cluster (see Figure 1).

\section{Discussion}

The aforementioned bowshock-like feature has been identified in $3.6 \mathrm{~cm}$ Very Large Array (VLA) multi-epoch observations of the central cluster by Zhao et al. (2009). They refer to the source as X24 and measure a proper motion of $V_{\mathrm{t}}=244 \pm 43 \mathrm{~km} \mathrm{~s}^{-1}$ and position angle $=-147^{\circ} \pm 9^{\circ}$, measured east of north. The radial velocity of $\mathrm{X} 24$ were found to be $V_{\mathrm{lsr}}=-18 \pm 8 \mathrm{~km} \mathrm{~s}^{-1}$.

Stellar bow shocks are caused by the interaction of a supersonic stellar wind with the surrounding medium. For a bow shock caused by an isotropic stellar wind, the standoff distance (the distance between a star and the tip of its bow shock along the symmetry axis) can be described in terms of the star's wind parameters (mass-loss rate and wind velocity), the density of the medium and the relative velocity between the star with respect to its surroundings (Wilkin 1996). 


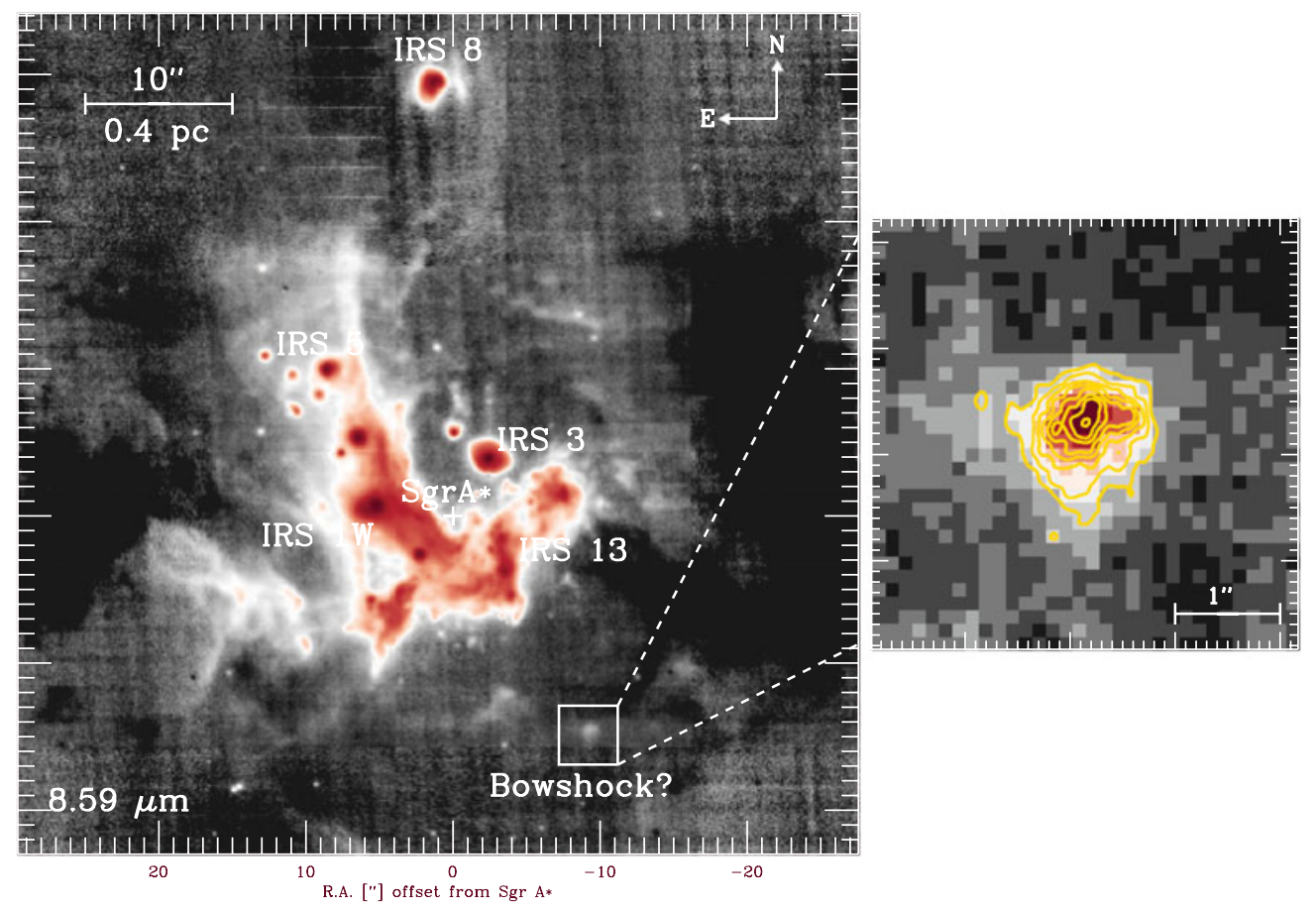

Figure 1. MIR mosaic of the central stellar cluster. Source X24 is shown as an inset to the right of the mosaic. [A COLOR VERsion is AVAilable onLine.]

Several observational arguments support the presence of an outflow originating from the inner portions of the Galactic center (Mužić et al. 2010, Markoff 2010, Crocker et al. 2011). The alignment of our feature with two previously found bow shock sources (marked by X7 and X3; see Mužić et al. 2010) and the minicavity may imply that all four features are produced by the same mechanism/event. All of these objects are aligned perpendicular to the plane of young mass-losing stars very close to the central SMBH. We estimate the velocity of a collimated flow that can cause such features to be $\sim 600-900 \mathrm{~km} \mathrm{~s}^{-1}$. It agrees with the estimates for the asymptotic speed of the nuclear star cluster's wind $\left(\sim 1000 \mathrm{~km} \mathrm{~s}^{-1}\right)$. Our value is slightly lower than the speed reported in Mužić et al. (2010), a possible indicator of mass loading between $\sim 0.06 \mathrm{pc}$ and $\sim 0.68 \mathrm{pc}$.

Although, by any astronomical standards, Sgr A* is not an active black hole, however, there is a growing body of evidence for the presence of an outflow from the material accreting onto the SMBH (Wang et al. 2013) or even indications for a jet (Yusef-Zadeh et al. 2012). The interaction of such an outflow with the ISM and wind of massive stars would produce signatures similar to our observations. The lack of a larger number of similar objects in the field can be explained either by the short life span of massive stars or the intermittent nature of the responsible mechanism (Sabha et al. in prep.).

\section{References}

Crocker, R. M., Jones, D. I., Aharonian, F., Law, C. J., Melia, F., Oka, T., \& Ott, J. 2011, MNRAS 413, 763

Markoff, S. 2010, PNAS 107, 7196

Mužić, K., Eckart, A., Schödel, R., Buchholz, R., Zamaninasab, M., \& Witzel, G. 2010, A\&A $521, \mathrm{~A} 13$ 
Tanner, A., Ghez, A. M., Morris, M. R., \& Christou, J. C. 2005, ApJ 624, 742

Viehmann, T., Eckart, A., Schödel, R., \& Pott, J.-U., Moultaka J. 2006, ApJ 642, 861

Wang, Q. D., Nowak, M. A., Markoff, S. B., Baganoff, F. K., Nayakshin, S., Yuan, F., Cuadra, J., Davis, J., Dexter, J., Fabian, A. C., Grosso, N., Haggard, D., Houck, J., Ji, L., Li, Z., Neilsen, J., Porquet, D., Ripple, F., \& Shcherbakov, R. V. 2013, Science 341, 981

Wilkin F. P. 1996, ApJL 459, L31

Yusef-Zadeh, F., Arendt, R., Bushouse, H., Cotton, W., Haggard, D., Pound, M. W., Roberts, D. A., Royster, M., \& Wardle, M. 2012, ApJL 758, L11

Zhao, J.-H., Morris, M. R., Goss, W. M., \& An, T. 2009, ApJ 699, 186 